\title{
Preparation of Low Molecular Weight Chitosan by Complex Enzymes Hydrolysis
}

\author{
Huafei Xie \\ Institute of Green Chemical Engineering, Zhongkai University of Agriculture and Engineering \\ Guangzhou 510225, China \\ Tel: 86-20-3417-2871Ｅ-mail: zhonghua2344@yahoo.com.cn \\ Zhengyu Jia (Corresponding author), Jinhui Huang \& Cuirong Zhang \\ Institute of Green Chemical Engineering, Zhongkai University of Agriculture and Engineering \\ Guangzhou 510225, China \\ Tel: 86-20-3417-2871Ｅ-mail: gdjiazhenyu@163.com
}

Received: January 20, 2011 Accepted: March 2, 2011 doi:10.5539/ijc.v3n2p180

This research was financially supported by National Science Foundation of China (20776164) and Key Scientific and Technological Projects of Guangdong Province (2010B011000007).

\begin{abstract}
Through the determination of viscosity average molecular weight, enzyme activity, hydrolysis ratio and reducing sugar concentration, the optimal technological conditions of complex enzymes composed of commercial $\alpha$-amylase, cellulase and pectinase were investigated. Results indicated that complex enzymes could result in low molecular weight chitosan ranged from 1000 to 4000 after enzymatic degradation for $2 \mathrm{~h}$ under the condition of zymolyte ratio $1: 5(\mathrm{~m} / \mathrm{m}), \mathrm{pH} 5.3$ and temperature $56^{\circ} \mathrm{C}$, and the structure of those products failed to vary obviously by FTIR analysis.
\end{abstract}

Keywords: Chitosan, Low molecular weight chitosan, Complex enzymes, Preparation

Chitosan (chitosan, CTS) is a kind of natural polysaccharide bioactive substances containing nitrogen, tends to be the sixth largest life elements excluding the body necessities viz. sugar, protein, fat, fiber and minerals, and exerts an extensive and intensive application in food, cosmetic and pharmaceutical areas (Zhu, 2006, PP. 41-45; Jiang, 2006, PP. 99-102). Due to its large molecular weight and the structure containing abundant hydrogen bonds, chitosan is only soluble in dilute acid, insoluble in water, and tough to be assimilated by human bodies, which hinders its application dramatically. If chitosan was degraded into LMWC (low molecular weight chitosan) with the molecular weigh less than 10000 , its water solubility increased greatly, conducive to human intestinal digestion and absorption, and possessed the functions such as promoting to produce splenic antibody, and lowering cholesterol, blood pressure, blood sugar and blood lipid level in serum and liver (Wei, 2003, PP. 614-617; Kim, 2005, PP. 357-368).

Recently, studies on the preparation of water soluble LMWC have attracted many researchers' attention all over the world (Huang, 2002, PP. 381-385). Degradation methods initiated by scholars mainly included chemical, physical and enzymatic degradation. Among them, enzymatic degradation has been recognized as one of the most promising approaches to prepare LMWC, because its reaction condition was moderate without byproducts and the degradation process was easy to monitor. However, the specific enzyme for chitosan was expensive, and its application in industry was limited. The applications of single non-specific enzyme in hydrolyzing chitosan such as $\alpha$-amylase (Yu, 2008, PP. 464-466; Zhang, 2003, PP. 44-48), pectinase (Ipsita, 2003, PP. 582-588; Cabrera, 2005, PP. 165-172), cellulose (Lin, 2009, PP. 47-53; Xia, 2008, PP. 6751-6762; Han, 2007, PP. 98-101;Xie, 2009, PP. 1895-1899), protease (Li, 2005, PP. 441-448) and so on have been documented, but those enzymes showed limited effects on degradation, even if enhancing their amount. Consequently, we investigated effects of complex enzymes containing cellulase, pectinase and $\alpha$-amylase on chitosan degradation. 


\section{Materials and methods}

\subsection{Materials}

Chitosan: Deacetylation Degree (DD) over 92\%, BR grade, Guangzhou Dongju experimental equipment Co., Ltd.; cellulase: 10u/mg, BR grade, Guangzhou Qiyun biochemistry technology Co., Ltd.; pectinase: $1000 \mathrm{u} / \mathrm{mg}$, BR grade, Guangzhou Qiyun biochemistry technology Co., Ltd.; $\alpha$-amylase: 3700u/g, BR-level, Beijing Star Obo Biotechnology Co., Ltd.; 3,5-dinitrosalicylic acid: BR grade, Sinopharm Chemical Reagent Co., Ltd.; other reagents were of AR grade.

\subsection{Equipment}

722 type visible-infrared spectrometer: Shanghai Precision Scientific Instrument Co., Ltd.; Ubbelohde viscometer: Shanghai Glass Instrument Factory; Precision pH meter: the Second Analytical Instrument Factory of Shanghai; Super constant temperature water bath: Changzhou Sino Instrument Co., Ltd.; Fourier transforms infrared spectrometer: PerkinElmer Company, USA; Vacuum drying oven: Gongyi Yingyu Yuhua Instrument factory.

\subsection{Experimental methods}

\subsubsection{Preparation of LMWC}

According to the reference(He, 2007, PP. 32-35), a certain amount of chitosan raw materials was dissolved in acetic acid-sodium acetate buffer with different $\mathrm{pH}$ values, and degraded in the manner of cellulose: pectin Enzyme: $\alpha$-amylase $(\mathrm{m} / \mathrm{m} / \mathrm{m})=1: 1: 1$. After reaction for certain time, $\mathrm{pH}$ was adjusted, enzymatic activity was inactivated for $10 \mathrm{~min}$ at $100{ }^{\circ} \mathrm{C}$, the resultant solution was filtered by membrane, and a certain amount of ethanol was added into supernatant. After standing for some time, the solution was filtered and the precipitation was undertaken vacuum drying.

\subsubsection{Determination of enzyme-hydrolyzed molecular weight}

Molecular weight was determined by viscometric method. $15 \mathrm{ml}$ supernatant after enzymatic inactivation was agitated rigorously, and added into Ubbelohde viscometer. At the constant temperature of $30^{\circ} \mathrm{C}$ in water bath, the lowering time of solvents and enzyme-hydrolyzed supernatant in Ubbelohde viscometer were measured by stopwatch. The average was obtained by multiple measurements. According to the method reported by the literature (Jiang, 2006, PP. 99-102), the intrinsic viscosity of hydrolysate ( $\eta$ ) was detected, and its viscosity average molecular weight $(\mathrm{M \eta})$ was calculated according to the following formula.

$$
[\eta]=11.0 \times 10^{-3} \mathrm{M}_{\eta}^{0.85}
$$

\subsubsection{Determination of reducing sugar concentration (Zhao, 2008, PP. 534-536)}

3, 5-dinitrosalicylic acid (DNS) method was applied in the determination. The standard curve was plotted by the absorbance of $\alpha$-amino-glucose at $520 \mathrm{~nm}$, and relative reducing sugar concentration was indentified according to the standard curve of hydrolysate $\triangle \mathrm{A} 520$.

\subsubsection{Determination of enzyme activity}

$1 \mathrm{~mL}$ appropriately diluted enzyme solution was added into $1 \mathrm{~mL} 0.02 \mathrm{~g} / \mathrm{mL}$ chitosan colloidal fluid and after agitation and reaction for $10 \mathrm{~min}$, the solution was heated by boiling water to inactivate enzymes. After cooling, $\mathrm{pH}$ value was adjusted from 7.0 to 8.0 , the solution was centrifuged at $4000 \mathrm{r} / \mathrm{min}$ for $10 \mathrm{~min}$. $\mathrm{A}_{520}$ was measured by DNS method using $1 \mathrm{~mL}$ supernatant, and reducing sugar concentration resulted from enzymatic degradation was obtained according to the standard curve. Complex enzyme activity was defined as follows: when complex enzyme degrade chitosan and release $1 \mu \mathrm{mol}$ relative reducing sugar at the condition of $\mathrm{pH} 5.3$ and $56^{\circ} \mathrm{C}$, and at that time, the consumed enzyme amount was recognized as an enzyme activity unit (U).

\subsubsection{Determination of chitosan hydrolysis (Chen, 2007, PP. 204-208)}

Complex enzyme was added into chitosann solutions, and agitated vigorously till even. After reaction at the optimum temperature for $2 \mathrm{~h}$, adjusting $\mathrm{pH}$ value to 8.0, centrifuging, the precipitation was rinsed till neutral, and the weight after drying $\left(\mathrm{W}_{1}\right)$ was measured. The inactivated complex enzyme was performed the same way, the weight after drying $\left(\mathrm{W}_{2}\right)$ was obtained. Hydrolysis rate was calculated in the following formula:

$$
\text { Hydrolysis rate }=\left(\mathrm{W}_{2}-\mathrm{W}_{1}\right) \times 100 / \mathrm{W}_{2}
$$

\subsubsection{IR analysis of hydrolysates (Zhang, 2003, PP. 44-48)}

Samples were assayed using KBr tablet by Fourier transform infrared spectroscopy. Deacetylation degree (DD) of products was calculated by the following formula: 


$$
\mathrm{DD}=\frac{1.33-\mathrm{A}_{1655} / \mathrm{A}_{3450}}{1.33} \times 100
$$

Where A1655 was the absorption peak of amide I at the wavelength of $1655 \mathrm{~cm}^{-1}$, and A3450 was the absorption peak of $\mathrm{NH}$ at the wavelength of $3450 \mathrm{~cm}^{-1}$.

\section{Results and discussions}

\subsection{Effects of temperature on complex enzymatic degradation}

According to the literatures, the optimum temperature for the enzyme activity of cellulase, pectinase and $\alpha$-amylase ranged from 40 to $60{ }^{\circ} \mathrm{C}$. In the present paper, effects of complex enzymes at the temperature ranging from 25 to $75{ }^{\circ} \mathrm{C}$ were investigated, and relative reducing sugar concentration of 6 different reaction solutions at different temperatures were measured respectively. Result was depicted in Figure 1. As seen from Figure 1, the optimum temperature for complex enzyme was $56{ }^{\circ} \mathrm{C}$, and when the temperature was below $56{ }^{\circ} \mathrm{C}$, increasing temperature could enhance enzyme degradation reaction rate; but on the other hand, because the nature of enzyme was protein, with the increasing of temperature, enzyme could inactivate gradually. Therefore, the optimum temperature for enzyme was the combined results of these two effects.

\subsection{Effects of $p H$ value on enzymatic degradation}

Solution $\mathrm{pH}$ value showed a great impact on enzymatic degradation of complex enzymes, and result was depicted in Figure 2. When $\mathrm{pH}$ value was over 6.5, the solubility of chitosan was poor, reaction occurred in a non-homogeneous system. In order to guarantee the reaction in the homogeneous condition, $\mathrm{pH}$ value was adjusted ranging from 4 to 6.5. As seen from Figure 2, the optimum $\mathrm{pH}$ value for complex enzyme to degrade chitosan was 5.3, when $\mathrm{pH}$ value was lower than 4.5 , enzyme activity was smaller; when $\mathrm{pH}$ value was higher than 5.5, enzyme activity began to decrease, which could be mainly ascribed to the fact that its natural components of enzyme, viz. protein, was an amphoteric electrolyte, $\mathrm{pH}$ value could affect not only the stability of enzyme molecular structure, but also the relevant groups dissociation and chitosan dissolution and ionization in its activity center, and thus affected the combination of enzyme and chitosan and change enzyme activity. Accordingly, only in the optimum $\mathrm{pH}$ value of 5.3, effects of complex enzymes on chitosan degradation was the most efficacious.

\subsection{Effects of zymolyte ratio on enzymatic degradation}

During the period of the experiment, the concentration of chitosan was $3 \%(\mathrm{~m} / \mathrm{v})$, enzyme concentration ranged from $0.15 \%$ to $1.5 \%$, and degradation time was $1 \mathrm{~h}$ for all treatments. Relative reduce sugar concentration was observed, and result was depicted in Figure 3. When zymolyte concentration was certain, with the increasing of enzyme amount, relative reducing sugar concentration increased; but when the added enzyme amount exceeded $0.6 \%$, relative reducing sugar concentration failed to increase obviously. After analysis, we speculated that complex enzyme acted on $\beta-(1,4)$ glycosidic bond of chitosan molecular chain at random in the manner of interior contact to degrade, and with the increasing of enzyme concentration gradually, the probability of binding and catalysis of $\beta-(1,4)$ glycosidic bond also increased. At the same degradation time, catalyze reaction in chitosan molecular occurred more frequently, and the amount of complex enzyme exceeded $0.6 \%$, its catalytic effect was tough to exert. Consequently, the more suitable added concentration should be $0.6 \%$.

\subsection{Effects of time on enzymatic degradation}

Chitosan and complex enzyme were added into chitosan acetic acid-sodium acetate buffer at $\mathrm{pH} 5.3$ in order to react, samples were selected to measure absorbance and intrinsic viscosity every certain time, and relative reducing sugar concentration and viscosity average molecular weight were calculated. Result was depicted in Figure 4. During the initial stage of reaction, relative reducing sugar concentration increased progressively, and viscosity average molecular weight decreased rapidly. When reaction continued to $2 \mathrm{~h}$, relative reducing sugar concentration and viscosity average molecular weight varied slowly, which could be ascribed to the fact that the amount of $\beta-(1,4)$ glycosidic bond existed within chitosan began to decrease while production concentration increased or reverse reaction enhanced. The reduction of enzyme activity also inhibited the degradation reaction to some extent, and thus had inhibitory impact on enzymatic reaction.

\subsection{IR spectrum}

Through IR spectra, we could determine the configuration of glycosidic bond and substitution of hydroxyl and amino groups (Wang, 2008, PP. 147-148). KBr tablet of raw chitosan and LMWC resulted from enzymatic degradation was scanned from 450 to $4000 \mathrm{~cm}^{-1}$. As seen from Figure 5, the IR spectra characterizations of raw 
chitosan and LMWC were basically consistent. Its characteristic absorption peaks, such as O-H stretching vibration at $3480 \mathrm{~cm}^{-1}, \mathrm{C}-\mathrm{H}$ stretching vibration at $2880 \mathrm{~cm}^{-1}$, amide I and II absorption peaks at $1650 \mathrm{~cm}^{-1}$ and $1555 \mathrm{~cm}^{-1}$ all existed, and peak area failed to vary significantly. Due to the increasing of hydroxyl group, strong $-\mathrm{OH}$ absorption band appeared at $3480 \mathrm{~cm}^{-1}$.

As DD level determined the quantity and types of amid groups in chitosan molecular involved in the formation of intramolecular and intermolecular hydrogen bonds, free amino absorption peak at about $1600 \mathrm{~cm}^{-1}$ reflected DD level. Before and after hydrolysis, the principal site and intensity of free amino bending vibration at about $3450 \mathrm{~cm}^{-1}$ and $1600 \mathrm{~cm}^{-1}$ didn't vary significantly, which further proved that such concerted reaction was triggered by the cracking of $\beta-(1,4)$ glycosidic bond, and elucidated that chitosan glycosidic ring structure was not destroyed before and after degradation. After calculation, DD has not changed.

\subsection{Mechanism of complex enzyme hydrolysis}

Chitosan degradation could be appropriately divided into two phases: because chitosan and cellulose are polysaccharide compounds linked by glycosidic bonds resulted from the polymerization of D-glucosamine, and due to quite similar structure, their degradation effects should be also similar. Interior contact played the cardinal role during the early period of hydrolysis, complex enzymes cut away the $\beta-(1,4)$ glycosidic bonds within chitosan molecular, chitosan solution viscosity decreased rapidly, molecular weight lowered from 1,000,000 to 100,000 , and the reaction didn't conform to Michealis-Menten kinetic equations. Due to the specifity of enzymes, effects of 3 enzymes on the degradation of $\beta-(1,4)$ glycosidic bonds within chitosan molecular began to decrease. After reaction for some time, cellulase, pectinase, and $\alpha$-amylase undertook selective cross-degradation on chitosan fragments obtained from degradation by different enzymes, and the molecular weight of chitosan lowered from 100,000 to 10,000. Reaction rate during this process was quite slow compared to the former phase. When oligomeric sugar concentration reached a certain level, they began to inhibit enzyme activities and limit degradation reaction, and at this time, enzymatic degradation terminated.

\subsection{Preparation technological line of LMWC by complex enzyme methods}

Based on the above results, we could obtain the optimum preparation technological line of LMWC by complex enzyme methods as follows:

According to the line, production rate reached more than $90 \%$ with good water solubility. Intrinsic viscosity of hydrolysates were depicted in Figure 6, and after calculation, average molecular weight of LMWC was all lower than 4000 .

\section{Conclusions}

3.1 Using complex enzymes composed of commercial cellulose, pectinase and $\alpha$-amylase, the optimum experimental parameters were temperature, $\mathrm{pH}$ value and zymolyte ratio as $53{ }^{\circ} \mathrm{C}, 5.3$ and $1: 5$, respectively. After reaction for about $2 \mathrm{~h}, \mathrm{LMWC}$ with the molecular weight of below 4000 could be obtained, and production yield reached more than $90 \%$ with good water solubility.

3.2 Complex enzymes could degrade the large molecular chain of chitosan rapidly under slight acidic conditions, decrease the viscosity average molecular weight, and tended to be the efficacious approach to prepare pharmaceutical LMWC.

3.3 The action mechanism of this complex enzymes cut down $\beta-(1,4)$ glycosidic bonds that linked D-glucosamine and D-acetyl-glucosamine at random in the manner of intrinsic contact, but didn't degrade one by one from the chain terminates.

3.4 Complex enzymes composed of commercial cellulose, pectinase and $\alpha$-amylase didn't change chitosan glycosidic ring structure and DD.

\section{References}

Cabrera, J.C., \& Custsem, P.V. (2005). Preparation of chitooligosaccharides with degree of polymerization higher than 6 by acid or enzymatic degradation of chitosan. Biochemical Engineering Journal, (25): 165-172.

Chen, X.E., Fang, X.B., \& Xia, W.S. (2007). Preparation of chitooligosaccharides by enzyme hydrolysis. Journal of Functional Polymers, 19(2):204-208.

Han, Y.P., \& Lin, Q. (2007). Preparation of oligochitosan by cellulase depolymerization with membrane technology. Chemical World, (2):98-101.

He, X.Y., \& Xia, W.S. (2007). Studies on enzymatic hydrolysis of chitosan. Food and Machinery, 23(3):32-35.

Huang, Y.C., Li, L., \& Guo, S.Y., et al. (2002). Progress of study on enzymatic hydrolysis of chitosan. Chemical 
Industry and Engineering Progress, 21(6):381-385.

Ipsita, R., Meryam, S., \& Munishwar, N.G. (2003). Hydrolysis of chitin by PectinexTM. Enzyme and Microbial Technology, (32): 582-588.

Jiang, T.D. (2006). Chitosan (the second edition). Beijing: Chemical industry press, 205-305, 99-102.

Kim, S.K., \& Rajapakse, N. (2005). Enzymatic production and biological activities of chitosan-oligosaccharides (COS): A review. Carbohydrate Polymers, (62):357-368.

Li, J., Du, Y.M., Yang, J.H., et al. (2005). Preparation and characterisation of low molecular weight chitosan and chitooligomers by a commercial enzyme. Polymer Degradation and Stability, (87):441-448.

Lin, S.B., Lin, Y.C., \& Chen, H.H. (2009). Low molecular weight chitosan prepared with the aid of cellulase, lysozyme and chitinase: characterisation and antibacterial activity. Food Chemistry, (116):47-53.

Wang, A.Q. (2008). Chitosan chemistry. Beijing: Science press, 147-148.

Wei, X.L., \& Xia, W.S. (2003). Research development of chitooligosaccharides physiological activities. Chinese Pharmacological Bulletin, 19(6):614-617.

Xia, W.S., Liu, P., \& Liu, J. (2008). Advance in chitosan hydrolysis by non-specific cellulases. Bioresource Technology, (99):6751-6762.

Xie, Y., Hu, J.G., \& Wei, Y., et al. (2009). Preparation of chitooligosaccharides by the enzymatic hydrolysis of chitosan. Polymer Degradation and Stability, (94):1895-1899.

Yu, H.Y., \& Wang, S. (2008). Study on enzymatic production of low chitosan. Food Science, 29(8):464-466.

Zhang, W.Q., Xia, W., \& Xu, H., et al. (2003). Reaction characteristics of chitosan hydrolysis catalyzed by non-specific enzymes. Journal of Functional Polymer, 16(1):44-48.

Zhao, K., Xu, P.J., \& Gu, G.Y. (2008). Study on measuring reducing sugar by DNS reagent. Food Science, 29(8):534-536.

Zhu G.Y., Qu, R.L., \& Li, F. (2006). Research progress in preparation and application of low molecular weight chitosan. Chinese Polymer Bulletin, (2):41-45.

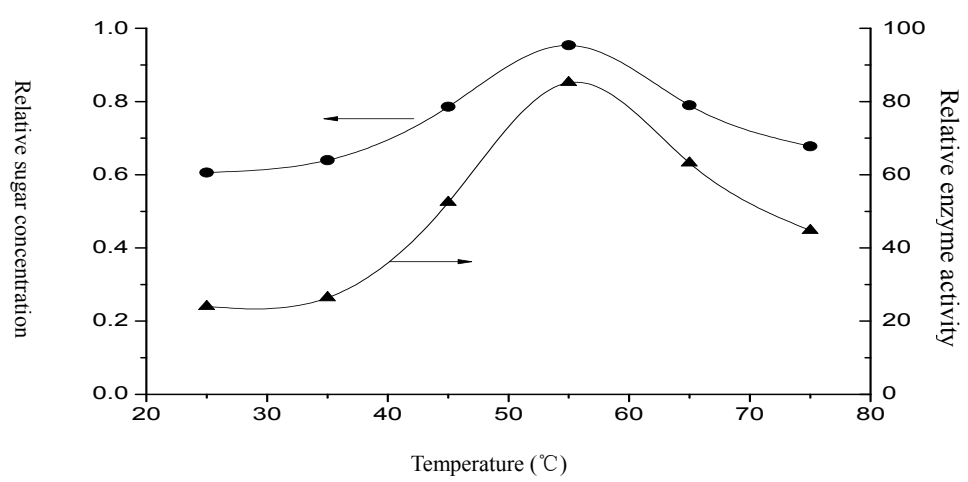

Figure 1. Effects of temperature on enzymatic degradation

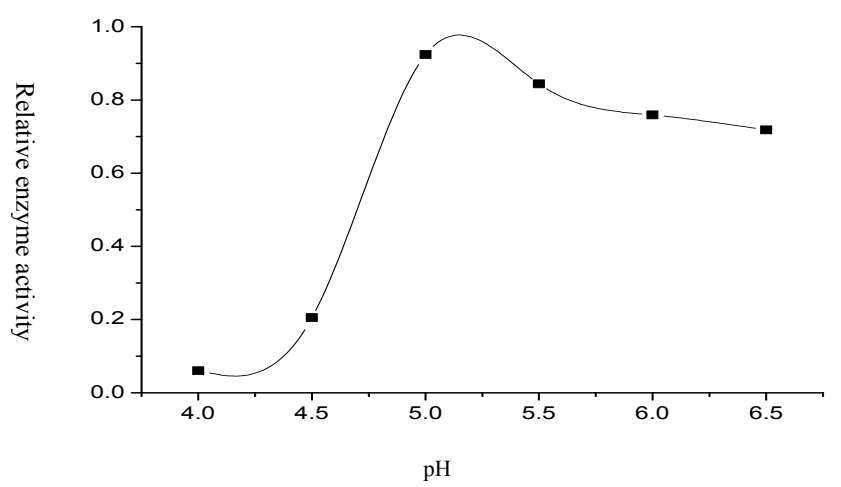

Figure 2. Effects of $\mathrm{pH}$ value on enzymatic degradation 


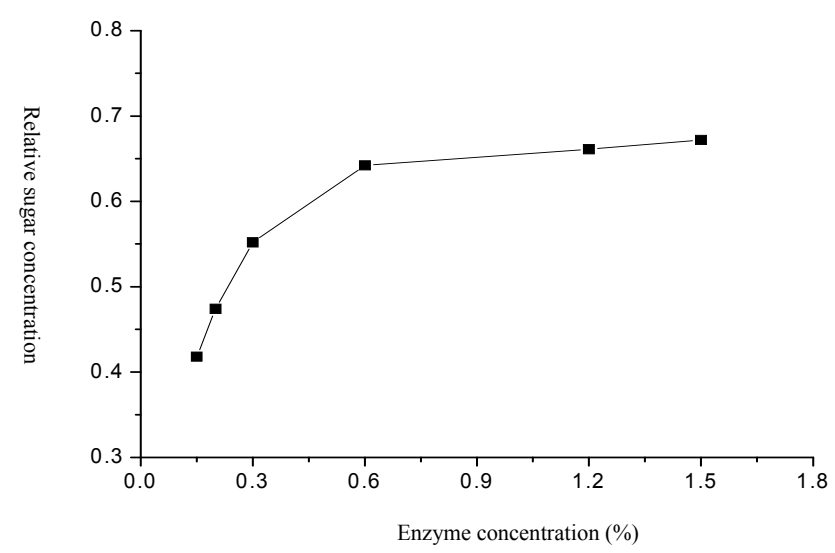

Figure 3. Effects of enzyme amount on enzymatic degradation
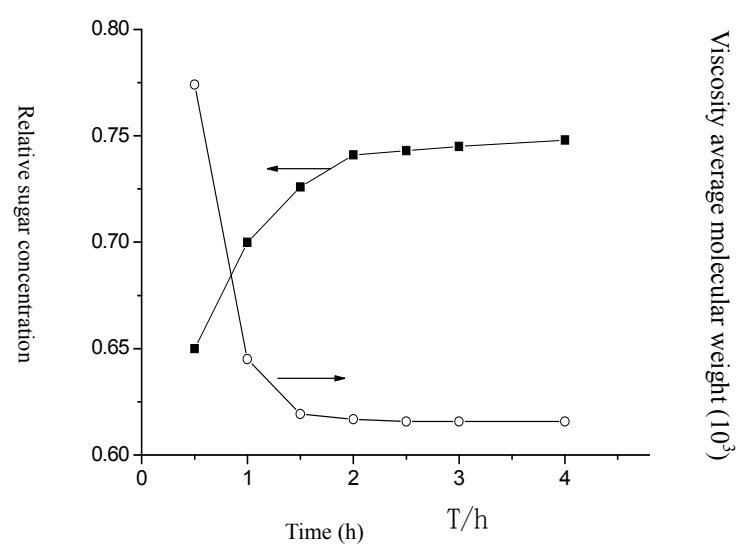

Figure 4. Effects of time on enzymatic degradation

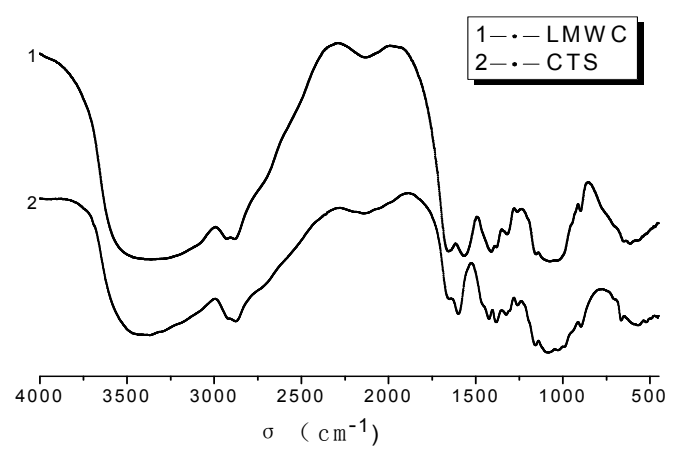

Figure 5. IR spectra of LMWC and CTS 


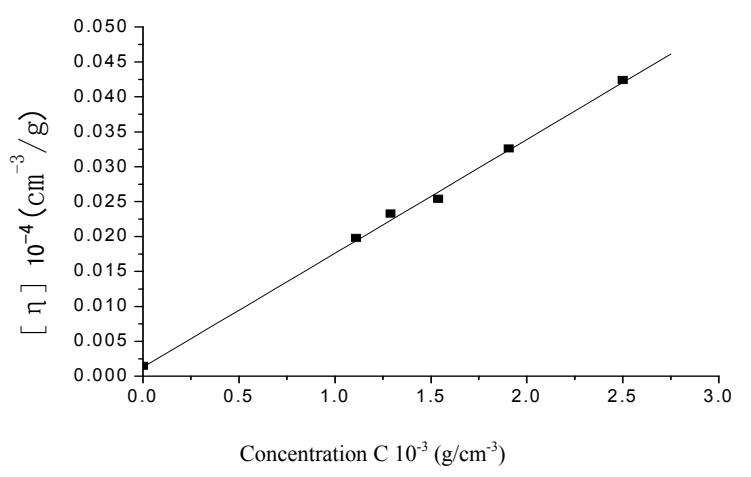

Figure 6. [ๆ]-C of LMWC intrinsic viscosity

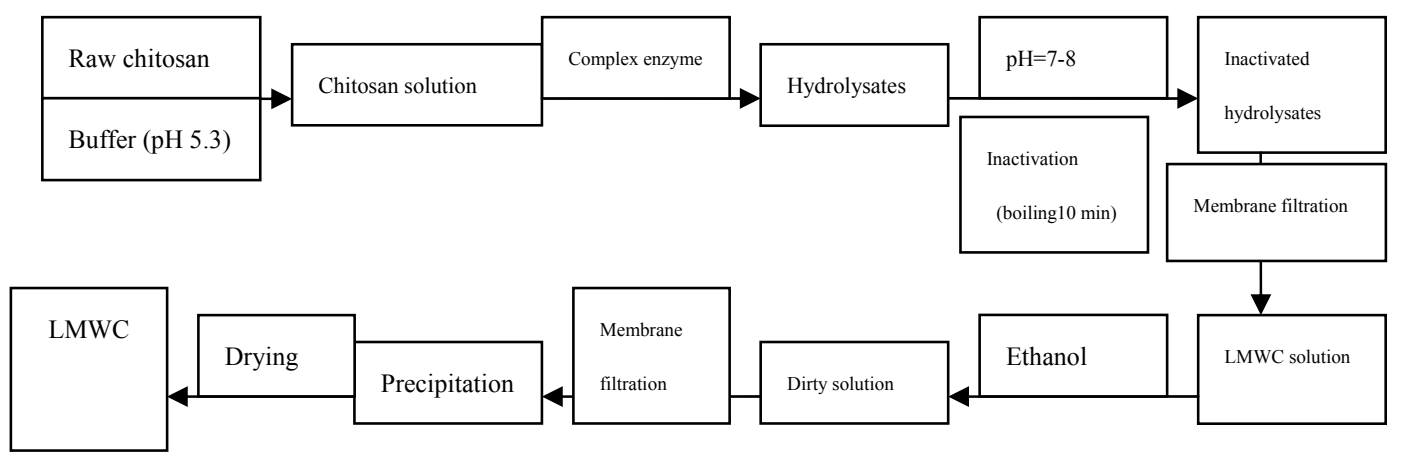

Figure 7. Preparation technological line of LMWC 